\title{
Monitoring plasmonic hot-carrier chemical reactions at the single particle level
}

\author{
Sabrina Simoncelli, (iD *ab Evangelina L. Pensa, (iD a Thomas Brick, ${ }^{a}$ \\ Julian Gargiulo, (D) a Alberto Lauri, (D) a Javier Cambiasso, (D) a Yi Li, (D) ac \\ Stefan A. Maier ${ }^{\text {ac }}$ and Emiliano Cortés (D) *ac
}

Received 30th September 2018, Accepted 24th October 2018

DOI: $10.1039 / \mathrm{c} 8 \mathrm{fd} 00138 \mathrm{c}$

\begin{abstract}
Plasmon excitation in metal nanoparticles triggers the generation of highly energetic charge carriers that, when properly manipulated and exploited, can mediate chemical reactions. Single-particle techniques are key to unearthing the underlying mechanisms of hot-carrier generation, transport and injection, as well as to disentangling the role of the temperature increase and the enhanced near-field at the nanoparticle-molecule interface. Gaining nanoscopic insight into these processes and their interplay could aid in the rational design of plasmonic photocatalysts. Here, we present three different approaches to monitor hot-carrier reactivity at the single-particle level. We use a combination of dark-field microscopy and photoelectrochemistry to track a hot-hole driven reaction on a single Au nanoparticle. We image hot-electron reactivity with subparticle spatial resolution using nanoscopy techniques. Finally, we push the limits by looking for a hot-electron induced chemical reaction that generates a fluorescent product, which should enable imaging plasmonic photocatalysis at the single-particle and single-molecule levels.
\end{abstract}

\section{Introduction}

Recent years have seen tremendous progress in the design of plasmonic antennas that can amplify and confine optical fields at the nanoscale. ${ }^{1}$ These optical antennas, fabricated with metallic nanostructures, convert free-space light into localized fields by coupling electronic-optical oscillations at the metal interface. The magnitude and spatial distribution of these high-field regions are very sensitive to the size, geometry and composition of the nanostructure. ${ }^{2}$ Indeed, these characteristics make them powerful tools for controlling external radiation

\footnotetext{
${ }^{a}$ The Blackett Laboratory, Department of Physics, Imperial College London, London SW7 2AZ, UK. E-mail: s. simoncelli@imperial.ac.uk

${ }^{b}$ Department of Physics, Randall Division of Cell and Molecular Biophysics, King's College London, London SE1 $1 U L, U K$

${ }^{c}$ Chair in Hybrid Nanosystems, Nanoinstitute Munich, Faculty of Physics, Ludwig-Maximilians-Universität München, 80799 München, Germany. E-mail: Emiliano.Cortes@lmu.de
} 
down to the sub-wavelength regime which, during the past two decades, has been exploited to modify the optical properties of nearby emitters, thus revolutionizing fields as diverse as bio-sensing and homeland security.

In addition to the opportunities presented by these optical antennas for controlling the photophysical processes of molecules, recent developments have also emerged via the combination of plasmonics and chemistry. ${ }^{3}$ The optical excitation of surface plasmons in metallic nanostructures leads not only to strongly enhanced light scattering, but also to enhanced light absorption. The non-radiative relaxation of the plasmon, which results in the generation of energetic charge-carriers (electron-hole pairs), can serve as an efficient source of energy for triggering chemical reactions. ${ }^{4,5}$ Unlike semiconductors, in which the properties of the carriers are determined by the band gap, plasmonic nanostructures allow for versatile manipulation. The tunability of nanostructure shape, size, composition and interface offers a high degree of control for tailoring the energy, spatial distribution and lifetime of the plasmon-derived hot-carriers. Fine control over these processes could assist in adapting the reactivity of plasmonic nanoparticles (NPs), thus providing exciting opportunities for photocatalysis. ${ }^{5}$ In this way, some of the pioneering studies in this area have demonstrated the possibility to plasmonically induce the water splitting reaction, ${ }^{6}$ the dissociation of $\mathrm{H}_{2}$ on $\mathrm{Au} \mathrm{NPs}^{7}$ or the epoxidation of ethylene and the oxidation of $\mathrm{CO}$ and $\mathrm{NH}_{3}$ on $\mathrm{Ag} \mathrm{NPs},{ }^{8}$ amongst other examples. ${ }^{9-12}$

Despite the growing interest and advances in the design of plasmonic nanoantennas that can be exploited to activate chemical transformations, many challenges still remain regarding their use as efficient hot-carrier injectors. The overall power conversion ultimately depends on the efficient extraction of the hot-carriers before thermalization and the subsequent regeneration of the surface charge density, either by restoring lost electrons or holes. ${ }^{12,13}$ In order to design efficient extraction schemes, much remains to be learnt about the energy and momentum distribution of the generated hot-carriers, the complex energy-transfer processes and the crucial role of the interface. ${ }^{\mathbf{1 4 - 1 6}}$ Furthermore, it is essential to disentangle the role of hot carriers from other phenomena linked to plasmon-excitation and decay that can also contribute to chemical transformations, such as increased temperature and near-field enhancement. ${ }^{17,18}$

Insights into the mechanistic details of the reactivity of hot-carriers have recently been unearthed by combining optical information on the plasmonic nanomaterials with ensemble kinetic analysis of the chemical transformations, either in the gas- or condensed-phase. ${ }^{19-21}$ While these ensemble schemes are a powerful source for helping to start uncover the complex interplay of processes controlling hot-carrier reactivity, they fail to address the role of the structural inhomogeneities of nanomaterials in their overall efficiency. ${ }^{22}$ Gaining nanoscopic insight at the single particle level and under in operando conditions is critical for guiding the efficient design and fabrication of reactive plasmonic platforms, i.e. for deciding the best shape, size, energy input, crystalline structure, reactive sites and material configuration (for multi-metallic materials or particlesubstrate combinations), among many other examples. ${ }^{23-26}$ However, pushing the current bulk-inspired methods down to the single-particle level presents the challenge of tracking chemical transformations of only a few thousand molecules. ${ }^{23,27,28}$ 
Here we present three different approaches for tracking and quantifying the reactivity of hot-carriers on single plasmonic nanoantennas. First, we explore a plasmonic hot-hole driven reaction under in operando conditions using a combination of dark-field microscopy and photoelectrochemistry. Second, we show that single-molecule based super-resolution schemes can be used to map hot-carrier reactivity beyond the diffraction limit of light, which we tested by visualizing the hot-electron driven desorption of thiol anchor groups from the surfaces of single Au nanostructures. Finally, we demonstrate the hot-electron driven reduction of Resazurin into Resorufin using Ag NPs. This fluorogenic reaction can open up new paths for revealing the nanoscopic aspects of hotcarrier driven reactions at the single-particle and single-molecule levels.

\section{Results}

\section{Hot-hole reactivity at the single particle level}

We combined electrochemistry and dark-field microscopy to study the role of a photoexcited Au NP in charge transfer processes, as schematized in Fig. 1a.

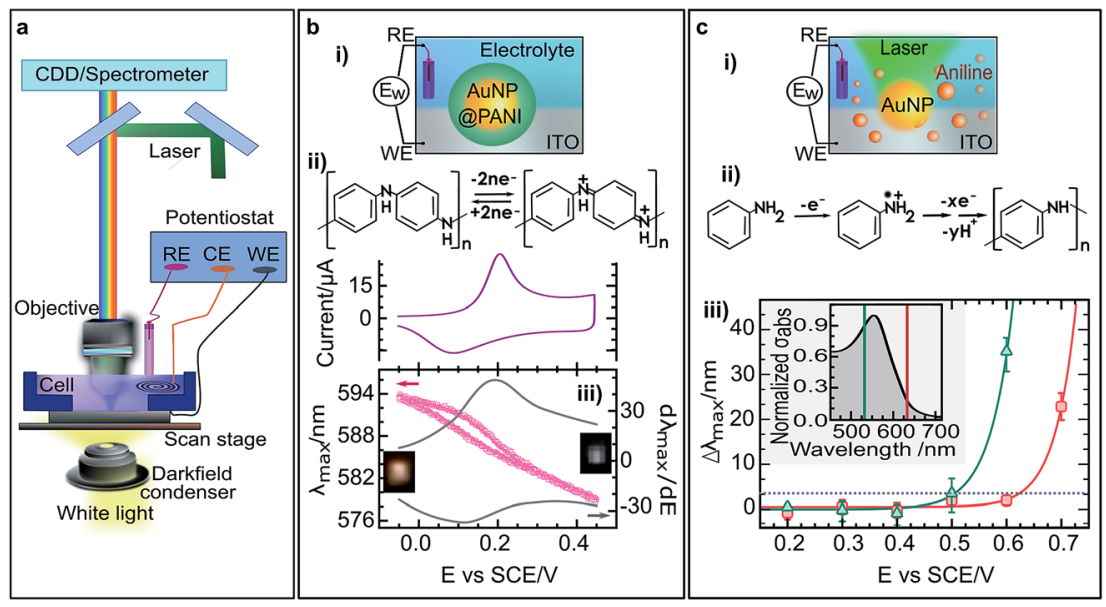

Fig. 1 Optoelectrochemical measurements of single Au NPs. (a) Scheme of the optoelectrochemical setup. (b) Monitoring the oxidation/reduction of the Au NP capping layer (polyaniline, PANI) at the single-particle level: (i) composition of the WE: PANI capped Au NPs (Au NPs@PANI) on ITO; (ii) cyclic voltammogram of PANI on polycrystalline Au. The upper inset image depicts the redox reaction; (iii) maximum scattering wavelength $\left(\lambda_{\max }\right)$ of a single Au NPQPANI as a function of $E$ (pink dots). The insets show dark-field images of a single Au NP@PANI at the two limit potentials. Through derivation of the spectroelectrochemical data it is possible to recover the redox profiles for PANI oxidation and reduction at the single particle level (grey solid lines). In all cases the applied potential was set between -0.05 to $0.45 \mathrm{~V}$ (vs. SCE) at $0.05 \mathrm{~V} \mathrm{~s}^{-1}$. (c) Plasmon-assisted electropolymerization of aniline: (i) scheme of the WE: Au NPs drop-casted on ITO. The reaction is conducted in aniline solution and under CW laser illumination; (ii) simplified aniline polymerization mechanism highlighting that radical formation is the rate-limiting step; (iii) red-shift in the $\lambda_{\max }\left(\Delta \lambda_{\max }\right)$ after the Au NPs were illuminated for $30 \mathrm{~s}$ at a given potential $E$ with either $532 \mathrm{~nm}$ (green triangles) or $633 \mathrm{~nm}$ (red squares) CW lasers. The irradiance for each laser wavelength was chosen such that the power absorbed by the Au NPs was the same. 
Electrochemical control was performed using a potentiostat and a three-electrode cell configuration using $0.5 \mathrm{M} \mathrm{H}_{2} \mathrm{SO}_{4}$ aqueous as supporting electrolyte. The saturated calomel electrode (SCE) and Pt coil served as the reference electrode (RE) and counter electrode (CE), respectively. The working electrode (WE) consisted of $80 \mathrm{~nm} \mathrm{Au}$ NPs drop-casted on ITO. The three-electrode electrochemical cell was mounted on a dark-field microscope with a water immersion objective. This optoelectrochemical setup allowed for the tuning of the WE electron's energy as well as the illumination and spectroscopic characterization of single nanoparticles. ${ }^{29}$

As a first example of the capabilities of our setup, we monitored the changes in the optical properties of a single Au NP when the electro-oxidation and electroreduction of its capping layer took place. As shown in Fig. 1b(i), the WE consists of Au NPs capped with polyaniline (PANI) and drop-casted on a ITO substrate. Fig. $1 \mathrm{~b}$ (ii) shows that the redox state of PANI can be switched by the applied potential, as depicted by the cyclic voltammogram curve of the system and the inset scheme of the reaction. As the potential $(E)$ increases linearly from -0.05 to $0.45 \mathrm{~V}$, the Au NPs drain electrons from the PANI layer, thus oxidizing it. As a result of the charge transfer process the current increases, peaking at $0.2 \mathrm{~V}$. It should be noted that the final state (at $0.45 \mathrm{~V}$ ) exhibits a higher current value than the initial state (at $-0.05 \mathrm{~V}$ ), which is characteristic of a more conductive PANI layer. ${ }^{30}$ When reversing the scan from $0.45 \mathrm{~V}$ to $-0.05 \mathrm{~V}$, the Au NPs inject electrons into the PANI layer and a reduction peak is observed at $0.1 \mathrm{~V}$. It must be noted that the measured current is an ensemble property corresponding to the total number of Au NPs (acting as the WE) on the ITO substrate. In order to monitor the process at the single-particle level we turned to following the optical changes (i.e. the scattering spectra) of a single Au NP whilst carrying out the electrochemical scan.

It has been shown that the dielectric function of PANI drastically changes depending on its redox state, being lower for the oxidized state. ${ }^{31}$ Therefore, the optical properties of Au NPs coated with PANI are expected to change when performing a cyclic voltammetry scan similar to that reported in Fig. $1 \mathrm{~b}(\mathrm{ii}) .^{32}$ Following the approach developed by the Landes group,,$^{33}$ we performed a singleparticle electrochemical experiment, but in this case we monitored the redox changes in the PANI capping layer through the optical response of a single Au NP. We used dark-field microscopy to localize and track the changes at the single particle level. Dark-field scattering spectra of a single PANI-capped Au NP (PANI@Au NP) were recorded as the potential was cyclically scanned between -0.05 and $0.45 \mathrm{~V}$. Fig. $1 \mathrm{~b}$ (iii) shows that the Au NP maximum scattering wavelength $\left(\lambda_{\max }\right)$ blue-shifts when the potential is increased and red-shifts when the potential is decreased (see the pink curve). The non-linear response and the hysteresis observed for $\lambda_{\max } v s$. $E$ - when comparing the direct and reverse electrochemical scans - reveal a non-reversible redox behaviour for the PANI layer of this Au NP (i.e. the PANI oxidation and reduction peaks are centered at different potentials). This can be extracted further when calculating the derivative of the scattering peak against the applied potential $\left(\mathrm{d} \lambda_{\max } / \mathrm{d} E\right)$, as shown in the grey curves of Fig. 1b(iii). In this way, it is possible to determine the electrochemical response of a single Au NP from the optical changes in its scattering spectra. ${ }^{33}$ This is further reinforced by the similarity between the electrochemically- 
measured cyclic voltammogram for many Au NPs (Fig. 1b(ii)) and the opticallyextracted redox behaviour of a single Au NP (Fig. 1b(iii)).

Taking advantage of the single-particle sensitivity and the precise electrochemical control of the WE electron's energy, we finally studied the electrooxidation of aniline on an optically excited Au NP. This configuration could assist in revealing the quanta of energy supplied by the illuminated $\mathrm{Au} \mathrm{NP}$ in order to drive the oxidation process; we should be able to monitor changes in the potential required to drive the reaction if there is any photocatalytic contribution from the Au NP. To this end, Au NPs were drop-casted on ITO and immersed in an aniline solution (Fig. 1c(i)). The potential was step-increased by $0.1 \mathrm{~V}$ from 0.2 to $0.7 \mathrm{~V}$, as an oxidation reaction needs to take place as shown in the diagram of Fig. 1c(ii).

For each applied potential, the Au NP was excited for 30 seconds of illumination with a diffraction-limited CW laser coupled through a water immersion objective (see Fig. 1a). Two laser lines were employed: $532 \mathrm{~nm}$ and $633 \mathrm{~nm}$. This allowed us to explore two nearly extreme situations: the maximum and minimum of the absorption spectrum for the $80 \mathrm{~nm} \mathrm{Au} \mathrm{NP}$ (see the inset spectrum in Fig. 1c(iii)). The laser irradiances were set at $2.2 \mathrm{~mW} \mathrm{~cm}^{-2}$ and $9.3 \mathrm{~mW} \mathrm{~cm}^{-2}$ for $532 \mathrm{~nm}$ and $633 \mathrm{~nm}$, respectively. These values guarantee that the absorbed power, and therefore the increase in temperature, is the same for both wavelengths. Fig. 1c(iii) shows the shift in the $\lambda_{\max }\left(\Delta \lambda_{\max }\right)$ as a function of the applied potential after single $\mathrm{Au}$ NPs were subjected to laser illumination. The green triangles correspond to a $\mathrm{Au} \mathrm{NP}$ illuminated at $532 \mathrm{~nm}$ while the red squares correspond to $633 \mathrm{~nm}$ illumination. In both cases, after a certain potential is reached, a drastic red-shift in the scattering peak is observed. These changes in the scattering spectra are due to PANI formation around the Au NP. Interestingly, the polymerization of aniline starts at different potentials for both irradiation wavelengths. When irradiated at $532 \mathrm{~nm}$, a red-shift of $\Delta \lambda_{\max }=3.4 \mathrm{~nm}$ is observed at $0.50 \mathrm{~V}$, while at $633 \mathrm{~nm}$ irradiation the same red-shift is reached at $0.62 \mathrm{~V}$ (see the dotted line in Fig. 1c(iii)). This result is even more surprising when considering that the absorbed power is the same for both wavelengths and thus the increase in temperature is the same. Aniline being a transparent molecule in the visible regime also rules out any possible near-field contribution or excitation. ${ }^{18}$ Hence, these results suggest that the electrochemical reaction is plasmoncatalyzed by the generated hot-holes on the Au NP and that the energy of the available holes depends on the excitation wavelength of light.

These results show that photoelectrochemical measurements can serve as a way to estimate the energy and efficiency of plasmon generated hot-carriers in single particles, thus revealing unknown aspects of the light-induced electron transfer mechanism. We then went on to explore the spatial reactivity of single plasmonic photocatalysts.

\section{Hot-electron reactivity-mapping at the single-particle level}

In recent years, single-molecule based super-resolution fluorescence microscopy techniques have emerged as a versatile method for mapping molecular targets with nanometer precision (i.e. beyond Abbe's diffraction limit of light). ${ }^{34}$ By separating the fluorescence emission of individual fluorophores in time using photo-conversion, photo-switching or transient binding approaches, these 
techniques can provide high resolution information thanks to the subsequent determination of the single fluorophore's position. ${ }^{35}$ Borrowing these ideas, we developed a nanoscopic approach that enables the imaging of reactive sites in plasmonic photocatalysts.

Fig. 2a illustrates the concept of our imaging approach, named metallic DNAPAINT (m-PAINT). ${ }^{36,37}$ DNA-PAINT (DNA-Points Accumulation for Imaging in Nanoscale Topography), ${ }^{38}$ is a recently developed localization based superresolution nanoscopy technique that uses single-stranded fluorescent-labelled DNA probes (imager strands) which transiently bind and quickly dissociate from their complementary DNA targets (docking strands) to reconstruct superresolved images. In m-PAINT, we combined this technique with single-particle femtosecond illumination to map the plasmon-induced hot-electron active sites on the surfaces of metallic nanomaterials. ${ }^{36,37}$

First, we chemically coupled DNA targets (docking strands) to the surfaces of the metallic nanostructures through a binder ligand. Mindful design of the binder ligand is of paramount importance to both: (i) achieve highly-resolved images and (ii) study different hot-electron reactions (i.e. by changing the functional anchor group of the binder or by incorporating an electron-acceptor molecule in it). In order to optimize the imaging capabilities of our technique, we set the docking strand height between the fluorophores and the metallic surface to $\sim 7 \mathrm{~nm}$, which diminishes the spatial fluorescence quenching effects on the metals. ${ }^{39}$ In our case, this was achieved by introducing a hydrophilic PEG unit in the form of hexaethylene glycol between the docking strand and a mercaptohexyl linker. Additionally, we used a backfilling molecule (6-mercapto-1-hexanol, $\mathrm{MCH})$ to ensure a more compact monolayer and therefore prevent unspecific
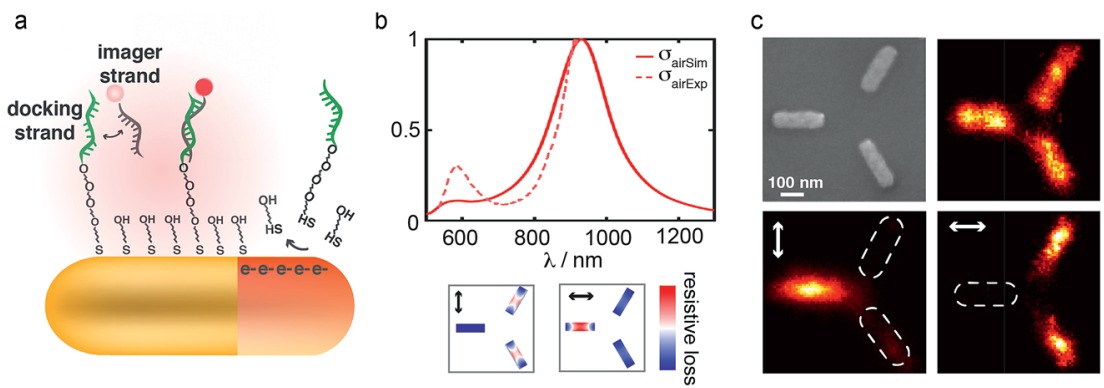

Fig. 2 Chemical-desorption mapping of thiolated-DNA molecules upon plasmonic hotelectron transfer. (a) Schematic of metallic DNA-PAINT. Docking strands (thiolated-DNA) are immobilized onto the Au surface of the plasmonic element providing transient binding sites for the complementary fluorescent-labelled DNA strands (imager strands). Upon plasmon excitation and hot-electron transfer, some docking strands are desorbed from the surface. (b) Optical performance of the Au nanorod trimer antenna. Each nanorod has a dimension of $180 \times 55 \mathrm{~nm}^{2}$. Top: Calculated (solid line) and experimental (dashed line) scattering cross-sections. Bottom: Near-field maps of the absorbed power in water (or resistive loss maps) for vertical or horizontal polarizations at $950 \mathrm{~nm}$ excitation. (c) Scanning electron microscope image of the plasmonic structure. Super-resolution $\mathrm{m}$ PAINT images for the non-irradiated sample and for the vertical/horizontal polarization excitation with $220 \mathrm{fs}$ laser illumination at $950 \mathrm{~nm}$ wavelength. Dashed curves indicate the borderline of the Au elements where thiol desorption has taken place (i.e. there is nobinding of the imager strand). 
binding of DNA strands on the surface of the metallic nanostructure. ${ }^{40}$ Our experimental approach allowed the imaging of single Au nanorod trimer structures with nanometric resolution (Fig. 2c, top right), perfectly agreeing with the obtained scanning electron microscope (SEM) images (Fig. 2c, top left).

Next, we studied the hot-electron driven cleavage of Au-sulfur bonds using a Au nanorod trimer system. Fig. $2 \mathrm{~b}$ (top) shows the scattering spectrum of the system in air. A plasmonic dipolar resonance is present at $910 \mathrm{~nm}$, corresponding to the resonance of each individual rod. At this wavelength, the scattering spectrum of the system is independent of the excitation polarization (data not shown). However, the spatial distribution of the non-radiative decay that governs the generation of hot electrons can largely differ between sub-elements depending on light polarization. ${ }^{36}$ Fig. $2 \mathrm{~b}$ (bottom) depicts the near-field power absorption maps (also named as resistive loss maps in the literature) for the two polarizations. We illuminated the system using a linearly polarized $950 \mathrm{~nm}$ femtosecond laser. By spinning the polarization of incident light, it is possible to selectively excite either the horizontal rod or the two diagonal rods. Fig. 2c (bottom) shows m-PAINT images of the trimers after irradiation. The dark areas of the antennas indicate the places where photocleavage has occurred, while the bright areas indicate the remaining binding domains after irradiation. The resemblance between the $\mathrm{m}$ PAINT maps and simulated absorption maps for both polarizations proves that the spatial distribution of energetic electrons responsible for triggering the $\mathrm{Au}-\mathrm{S}$ bond cleavage can be imaged with sub-diffraction resolution.

\section{Hot-electron reaction for tracking in operando single-photocatalyst activity}

This section aims to extend the study of hot-carrier reactions from the single photocatalyst to the single molecule level as they happen in situ. Single-molecule studies of single nanocatalysts have recently been implemented using fluorogenic reactions, ${ }^{28,41}$ where a non-fluorescent reactant is converted into a fluorescent product. One of the most employed of such reactions is the reduction of the nonfluorescent Resazurin (Rz) molecule when reacting with hydroxylamine on the surfaces of $\mathrm{Au}$ nanoparticles (heterogeneous catalysis) to produce the highly fluorescent Resorufin (Rs) molecule, ${ }^{28}$ as schematized in Fig. 3a. The high sensitivity of fluorescence allows for the detection and quantification of single turn-over events, which appear as spikes in the collected fluorescence signal, as shown in the inset of Fig. 3a. Following this approach, kinetics studies of several single heterogeneous nanocatalysts have been studied, revealing reaction pathways,${ }^{28}$ size $^{41}$ and facet dependence, ${ }^{42}$ sub-particle distribution of active sites,${ }^{34}$ or even activation energies. ${ }^{43}$

In order to extend this technique to the study of hot-carrier driven chemical reactions at the single photocatalyst and single molecule levels, it is crucial to identify a fluorogenic reaction driven by hot-carriers. Here, we show that Ag spherical nanoparticles (Ag NPs) illuminated at their plasmon resonance can produce hot-electrons capable of reducing Rz into Rs without the use of hydroxylamine or any other reducing agent, as schematized in Fig. $3 \mathrm{~b}$.

We carried out ensemble-level fluorescence measurements to study the photocatalysis of Rz to Rs in the presence of plasmonic $60 \mathrm{~nm} \mathrm{Ag}$ NPs. Fig. 3c shows the absorption spectra of these three components. Due to excitation of the plasmon resonance, the Ag NPs present an absorption maximum at $430 \mathrm{~nm}$. We 
a Heterogeneous Catalysis

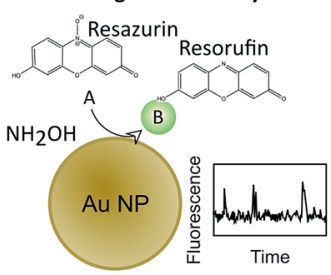

b Hot-carrier Photocatalysis

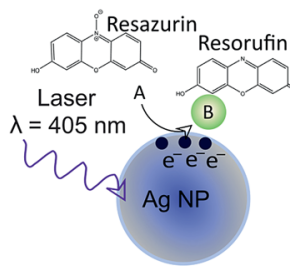

c

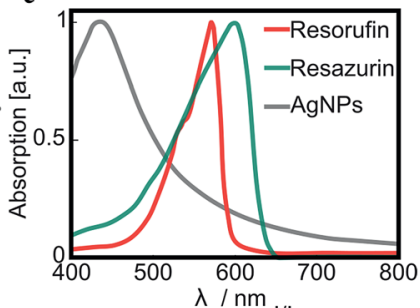

d
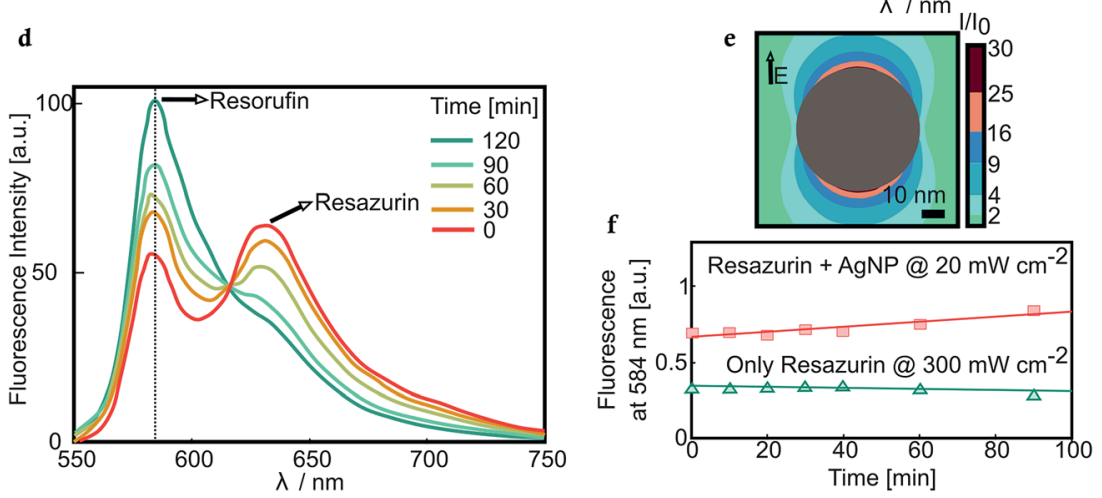

Fig. 3 (a) Example of a heterogeneous catalysis experiment. A weakly fluorescent molecule, $\mathrm{A}$, is converted into a highly fluorescent one, $\mathrm{B}$, on the nanocatalyst surface. Each turn-over event is detected as a spike in the fluorescence signal. (b) Working principle of hot-carrier driven photocatalysis. A plasmonic Ag NP is resonantly illuminated with a continuous wave laser at $405 \mathrm{~nm}$, generating highly energetic electrons. These electrons reduce the weakly fluorescent Resazurin molecule into the fluorescent Resorufin molecule. (c) Normalized absorption spectra of $60 \mathrm{~nm}$ Ag nanoparticles (grey), Resorufin (red) and Resazurin (green). (d) Photoconversion of an initial $5 \mu \mathrm{M}$ Resorufin solution (red) over increasing irradiation times. The spectra were normalized to the iso-emissive point at $613 \mathrm{~nm}$. The vertical dashed line indicates the Rs emission peak at $584 \mathrm{~nm}$ used in (f). (e) Map of the intensity enhancement produced by a $60 \mathrm{~nm}$ Ag sphere illuminated at $405 \mathrm{~nm}$. The polarization of the beam is indicated with an arrow. (f) Fluorescence intensity at the Resazurin peak (584 nm) vs. time for a solution containing $5 \mu \mathrm{M}$ Resazurin and $20 \mathrm{pM} \mathrm{Ag}$ NPs illuminated at $20 \mathrm{~mW} \mathrm{~cm}^{-2}$ (pink line), and for a solution of $5 \mu \mathrm{M}$ Resazurin without Ag NPs and with 15 times more power $\left(300 \mathrm{~mW} \mathrm{~cm}^{-2}\right.$ ) (green line). The curves have been vertically shifted for comparison.

illuminated a cuvette containing $20 \mathrm{pM} \mathrm{Ag} \mathrm{NPs} \mathrm{and} 5 \mu \mathrm{M}$ Rs with a $405 \mathrm{~nm} \mathrm{CW}$ laser at a power density of $300 \mathrm{~mW} \mathrm{~cm}^{-2}$. At this wavelength, the direct absorption of light by Rs or Rz is very low. Fig. 3d illustrates the variation in the fluorescence spectra for increasing irradiation times. Before irradiation (red curve), an emission peak at $634 \mathrm{~nm}$ corresponding to the weakly fluorescent $\mathrm{Rz}$ molecule could be observed. In addition, a small number of highly fluorescent Rs molecules contribute to an emission peak at $585 \mathrm{~nm}$. Dye conversion can be followed through the increase and accompanying decrease in the fluorescence emission peaks of Rs and Rz, respectively. After 150 minutes, almost the entire solution is converted, with the final spectrum resembling that of Rs. Pertinent controls performed in the absence of Ag NPs or without illumination showed no significant conversion. These experiments effectively demonstrate that it is possible to photocatalyse the reduction of Rs into $\mathrm{Rz}$ when exciting the plasmon resonance of 
the Ag NPs. However, plasmonic NPs can catalyse chemical transformations due to enhanced fields, thermal effects, or by the generated carriers. ${ }^{5}$ In order to prove that this reaction can be used as a hot-electron probe, thermal and field induced conversion should be ruled out.

The temperature increase $\Delta T$ of an illuminated single Ag NP can be estimated using the equation $\Delta T=\frac{\sigma_{\mathrm{abs}} I}{4 \pi k a}$, where $\sigma_{\mathrm{abs}}$ corresponds to the absorption cross section, $I$ the irradiance, $k$ the thermal conductivity of the immersion media and $a$ the NP radius. ${ }^{44}$ Applying this function using values of $\sigma_{\text {abs }}=1.2 \times 10^{-14} \mathrm{~m}^{2}, k=$ $0.59 \mathrm{~W} \mathrm{~K} \mathrm{~m}^{-1}$ and $a=30 \mathrm{~nm}$, we envisage a negligible temperature increase on the order of just $10^{-4} \mathrm{~K}$. The above assumes an isolated nanoparticle, but recent studies have shown that collective effects for highly concentrated solutions can lead to a marked increase in the temperature of the solution, which is some orders of magnitude greater than that expected for isolated NPs. ${ }^{45}$ In order to rule out such macroscopic temperature rises a thermocouple probe was inserted into a solution of $20 \mathrm{pM} \mathrm{Ag}$ nanoparticles, and the solution was illuminated with a $405 \mathrm{~nm}$ laser at the maximum power intensity used in our experiments. No temperature increase was observed, meaning that the temperature increase was smaller than the sensitivity of the thermocouple $(0.1 \mathrm{~K})$.

Next, we investigated whether the conversion reaction can be attributed solely to the enhancement in the electric field around the NPs upon resonant illumination. Fig. 3e depicts finite difference time domain (FDTD) calculations of field intensity enhancements around a $60 \mathrm{~nm}$ diameter Ag NP suspended in water upon illumination at $405 \mathrm{~nm}$. Within a shell of $10 \mathrm{~nm}$ around the nanoparticle surface, the average field intensity over all three planes is 14.9 times greater than that of the incident wave. We can then rule out the notion that it is solely a field induced effect through a comparison of the dye conversion with and without nanoparticles, using a 15 times higher power density in the latter case (see Fig. 3f). This way, both systems exhibit the same magnitude of field intensity. Fig. $3 \mathrm{f}$ shows the fluorescence intensity at the emission peak of Rs as a function of time. In the absence of Ag NPs and with the use of a 15 times higher beam intensity (300 $\mathrm{mW} \mathrm{cm}^{-2}$ ), no clear increase in the fluorescence peak intensity is observed. For a lower beam intensity $\left(20 \mathrm{~mW} \mathrm{~cm}^{-2}\right)$ in the presence of $\mathrm{Ag}$ NPs, a slight but discernible increase in the peak intensity over time is observed, which is indicative of the conversion of $\mathrm{Rz}$ to Rs. The lower magnitude of this conversion relative to that illustrated in Fig. $3 \mathrm{~d}$ is to be expected given the low laser power involved.

The results presented here allow us to claim that the reduction of Rz to Rs is driven by hot-carriers induced upon the resonant excitation of plasmons in $\mathrm{Ag}$ nanoparticles. This result paves the way for the investigation of hot-carrier reactions at the single molecule level.

\section{Conclusions}

The rational design of plasmonic photocatalysts requires a deeper understanding of the mechanisms contributing to plasmon-induced chemical reactions. Singleparticle and single-molecule studies were carried out in order to decouple the ensemble effects, thus facilitating the interpretation of the experimental results and offering easier routes to improve the performance of the photocatalyst under 
in operando conditions. This has been exemplified in the present contribution through a study of the energy or spatial distribution of plasmonic hot-carriers in single plasmonic particles coupled to different reactive interfaces. Finally, we have demonstrated the reaction conditions that are required in order to drive the hot-electron conversion of a fluorogenic molecule, which could serve as a reactive probe for future single-molecule studies of a diverse number of super-resolution schemes.

\section{Experimental}

\section{Sample preparation for optoelectrochemical measurements}

All reagents were of high-purity and were used as received. For aqueous solutions, ultra-pure water was employed $\left(18.2 \mathrm{M} \Omega \mathrm{cm}\right.$, MilliQ $\left.{ }^{\circledR}\right)$. Au NPs (80 nm in diameter, capped with CTAB, OD solution: 0.05) were synthesized and purified according to the method reported by Yoon J. H. et al. ${ }^{46} \mathrm{Au}$ NPs@PANI were obtained via the chemical synthesis of PANI layers (19 $\mathrm{nm}$ in thickness) around the Au NPs, following the protocol described by Xing S. et al. ${ }^{47}$

\section{Sample preparation for hot-electron reactivity mapping}

Gold trimer nanorods with dimensions of $180 \times 55 \mathrm{~nm}^{2}$ were fabricated on borosilicate glass using electron beam lithography (E-line, Raith $\mathrm{GmbH}$ ). The substrate was first coated with a positive-tone poly(methyl methacrylate) (950 K A4) resist and then baked for $3 \mathrm{~min}$ at $180{ }^{\circ} \mathrm{C}$. The nanostructures were then defined by exposure to an electron beam, followed by a development procedure. Chromium and gold thermal evaporation at $1.5 \AA^{-1}$ (Amod, Angstrom Engineering Inc.) and a standard lift-off process at $70{ }^{\circ} \mathrm{C}$ completed the fabrication process. For DNA functionalization the samples were first left for $1.5 \mathrm{~min}$ in a UVO cleaner/oxidizer (Femto, Diener Electronic GmbH) and mounted onto a bottomless self-adhesive slide (sticky-Slide VI 0.4, Ibidi) in order to form a flow chamber with an inner volume of $\sim 30 \mu \mathrm{L}$. DNA functionalization was carried out via the overnight incubation at RT of a $1 \mu \mathrm{M}$ solution of the single stranded DNA docking strand (ThiolC6-SpacerC18-ATGAGTTAATT, biomers.net $\mathrm{GmbH}$ ) diluted in $10 \mathrm{mM}$ Tris-HCl, $1 \mathrm{M} \mathrm{NaCl}, \mathrm{pH}=7$ (buffer A). Next, the sample was incubated in a $1 \mathrm{mM}$ 6-mercapto-1-hexanol (MCH, Sigma Aldrich) solution (30 minutes, RT) prior to a high-volume wash with buffer A. Finally, the sample was left in a solution of $10 \mathrm{mM}$ Tris-HCl, $50 \mathrm{mM} \mathrm{NaCl}, \mathrm{pH}=7$ (buffer B) or $5 \mathrm{mM}$ Tris- $\mathrm{HCl}, 10 \mathrm{mM}$ $\mathrm{MgCl}_{2}, 1 \mathrm{mM}$ EDTA, $\mathrm{pH}=8$ (buffer $\mathrm{C}$ ) for use in femtosecond pulsed laser irradiation or fluorescence imaging, respectively, after washing with buffer B.

For the scanning electron microscopy imaging, a $20 \mathrm{~nm}$ thin film of Espacer $300 \mathrm{Z}$ was coated onto the sample before DNA functionalization at $2000 \mathrm{rpm}$ for $60 \mathrm{~s}$, followed by a $60 \mathrm{~s}, 90{ }^{\circ} \mathrm{C}$ bake.

\section{Dark-field microscopy and spectroscopy}

Optical measurements for the optoelectrochemical configuration were carried out using a Witec microscope. For dark-field imaging, the sample was illuminated from the bottom with a white light source (OSL2, Thorlabs, United States) through an oil-immersion dark-field ultra-condenser (NA $=1.2-1.4$, Zeiss, Germany). Scattered light was collected using a water immersion objective $(\mathrm{NA}=1.0, \times 63$ 
magnification, Zeiss, Germany) and directed to a colour CCD camera. Scattering spectra were recorded before and after each Au NP was illuminated. Singleparticle dark-field scattering spectroscopy measurements of the gold nanorod trimers in air were carried out using an upright Nikon Eclipse Ti-U microscope (Nikon Instruments) equipped with a dark-field objective (Nikon LU Plan ELWD $100 \times$ NA 0.80 ), which is used to both provide the incident illumination and to collect the scattered light. For illumination, a $100 \mathrm{~W}$ halogen lamp (Nikon) was used. The scattered light was measured using a diffractive grating and a chargecoupled device camera cooled to $70 \mathrm{~K}$. The plotted spectra were corrected for the system wavelength response (by measuring the cross section of a perfect reference white rough scatter) as well as for the dark and background counts.

\section{Electrochemistry}

Electrochemical measurements were carried out at room temperature $24.0 \pm$ $0.2{ }^{\circ} \mathrm{C}$ ) in a three-electrode configuration cell and with a CHI760C potentiostat (CH Instruments, United States). A $0.5 \mathrm{M} \mathrm{H}_{2} \mathrm{SO}_{4}$ (99.99\%, Sigma-Aldrich) aqueous solution was used as the supporting electrolyte. Aniline (99\%, Sigma Aldrich) was dissolved in the supporting electrolyte until a final concentration of $0.2 \mathrm{M}$ was reached. The working electrode consists of $80 \mathrm{~nm}$ Au nanoparticles drop-casted on ITO (Nanoscribe, Germany). A Pt coil (99.99\%, Goodfellow, UK) and a saturated calomel electrode (SCE) served as the counter and reference electrodes, respectively. Before every experiment the Pt coil was cleaned using a butane flame annealing process followed by a quenching procedure in ultrapure water.

\section{Femtosecond pulsed-laser irradiation}

For the femtosecond pulsed-laser irradiation of the individual gold trimer nanorods a pulsed Yb:KGW PHAROS laser system was used as the pump of an ORPHEUS collinear optical parametric amplifier with a LYRA wavelength extension option (Light Conversion Ltd., pulse duration of $180 \mathrm{fs}$, repetition rate of 100 $\mathrm{kHz})$. The excitation beam $(\lambda=950 \mathrm{~nm})$ was reflected by a short-pass dichroic mirror (Thorlabs DMSP805) and focused onto a $\sim 1 \mu \mathrm{m}^{2}$ spot on the sample with a $40 \times$ (CFI S Plan Fluor ELWD $40 \times$, NA 0.60, Nikon) air objective. The beam polarization was adjusted using a half-wave plate and a polarizer, and the power fluence was set to $1.7 \mathrm{~mJ} \mathrm{~cm}{ }^{-2}$. The sample was fixed to an XYZ piezo-scanner stage (Nano-Drive, Mad City Laboratories), which allows for automatic single nanoantenna irradiation (10 seconds per antenna). Alignment of the antennas with the excitation beam was made possible by inspecting the sample with white light illumination and detecting the sample using a CCD camera (QICAM Fast 1394, QImaging).

\section{Fluorescence microscopy imaging}

Fluorescence imaging was carried out using an inverted Nikon Eclipse Ti-U microscope (Nikon Instruments). The excitation beam at $640 \mathrm{~nm}$ was provided by a $20 \mathrm{~mW}$ CW laser (LDH, Picoquant) spectrally filtered with a clean-up filter (BrightLine HC 636/8, Semrock). A single-edge dichroic beam splitter (Semrock BrightLine FF 649) was used to reflect the excitation light into the $100 \times, 1.49$ NA oil immersion Apo DIC N2 TIRF objective. The fluorescence light was spectrally filtered using an emission filter (ET705/72m, Chroma Technology) and collected 
onto an electron-multiplying charge-coupled device (EMCCD) camera (Evolve 512 Delta, Photometrics) operated at $100 \mathrm{~ms}$ per frame. For the DNA-PAINT imaging, a solution of the imager strand (Atto655-TTAACTCATG-3', biomers.net GmbH) diluted to $5 \mathrm{nM}$ in buffer $\mathrm{C}$ was added to the sample immediately before starting the measurements. 20000 frames per super-resolution image were recorded at an excitation intensity of $450 \mathrm{~W} \mathrm{~cm}^{-2}$.

\section{Super-resolution data processing and image analysis}

m-PAINT images were reconstructed from the raw data based on the subsequent localization of single molecules via Gaussian fitting using the rainSTORM Matlab application. Prior to single-molecule localization, stage drift correction and background subtraction of the photoluminescence (static, autofluorescence emission) from the gold trimer nanorods were carried out via the utilisation of an in-house Matlab routine. Images of the individual gold trimer nanorods were reconstructed from $\sim 700$ to 3000 localizations. Particle averaging of the gold trimer nanorods was carried out using a translation registration algorithm (Matlab built-in function). Average m-PAINT images were usually reconstructed by overlaying super-resolution images of 30 to 36 individual gold nano-antennas that had been exposed to the same chemical/optical conditions.

\section{Resazurin photoconversion}

Absorption spectra were recorded using a UV-Vis Agilent Technologies Cary 60 spectrometer. Fluorescence spectra were recorded using an Agilent Technologies Cary Eclipse Spectrophotometer, exciting at $520 \mathrm{~nm}$ and monitoring from 530-800 $\mathrm{nm}$.

A $10 \mathrm{~mm}$ optical path fused quartz cuvette was illuminated using a continuous wave Coherent Cube laser expanded through a telescope to a beam waist of $1 \mathrm{~cm}$, such that the entire solution was illuminated. After a determined irradiation time the cuvette was removed from the beam path and measured in the spectrometer.

\section{Numerical simulations}

Numerical calculations of the absorption and scattering cross-section spectra and the near-field intensity distributions were carried out using both Lumerical FDTD 2017a and Comsol Multiphysics v4.4 software. ${ }^{36}$

\section{Conflicts of interest}

There are no conflicts to declare.

\section{Acknowledgements}

This work has been supported by the EPSRC through the Reactive Plasmonics Programme (EP/M013812/1), the Royal Society and the Lee-Lucas Chair in Physics. S. S. acknowledges the financial support received from the Human Frontier Science Program Organization through a postdoctoral fellowship. J. G. acknowledges the financial support received from the European Commission through a Marie Skłodowska-Curie fellowship. Y. L. acknowledges the funding received from the European Union's Framework Programme for Research and 
Innovation Horizon 2020 (2014-2020) under the Marie Skłodowska-Curie Grant Agreement No. 754388 and from LMU Munich's Institutional Strategy LMU excellent within the framework of the German Excellence Initiative (No. ZUK22). E. C. acknowledges the financial support received from the European Commission through the ERC Starting Grant CATALIGHT (802989).

\section{References}

1 N. J. Halas, S. Lal, W.-S. Chang, S. Link and P. Nordlander, Plasmons in Strongly Coupled Metallic Nanostructures, Chem. Rev., 2011, 111, 39133961.

2 V. Giannini, A. I. Fernández-Domínguez, S. C. Heck and S. A. Maier, Plasmonic Nanoantennas: Fundamentals and Their Use in Controlling the Radiative Properties of Nanoemitters, Chem. Rev., 2011, 111, 3888-3912.

3 S. Linic, P. Christopher and D. B. Ingram, Plasmonic-metal nanostructures for efficient conversion of solar to chemical energy, Nat. Mater., 2011, 10, 911.

4 M. L. Brongersma, N. J. Halas and P. Nordlander, Plasmon-induced hot carrier science and technology, Nat. Nanotechnol., 2015, 10, 25.

5 E. Cortés, Efficiency and Bond Selectivity in Plasmon-Induced Photochemistry, Adv. Opt. Mater., 2017, 5, 1700191.

$6 \mathrm{~S}$. Mubeen, et al., An autonomous photosynthetic device in which all charge carriers derive from surface plasmons, Nat. Nanotechnol., 2013, 8, 247.

7 S. Mukherjee, et al., Hot Electrons Do the Impossible: Plasmon-Induced Dissociation of $\mathrm{H}_{2}$ on $\mathrm{Au}$, Nano Lett., 2013, 13, 240-247.

8 P. Christopher, H. Xin and S. Linic, Visible-light-enhanced catalytic oxidation reactions on plasmonic silver nanostructures, Nat. Chem., 2011, 3, 467.

9 T. Oshikiri, K. Ueno and H. Misawa, Selective Dinitrogen Conversion to Ammonia Using Water and Visible Light through Plasmon-induced Charge Separation, Angew. Chem., Int. Ed., 2016, 55, 3942-3946.

$10 \mathrm{X}$. Zhang, et al., Product selectivity in plasmonic photocatalysis for carbon dioxide hydrogenation, Nat. Commun., 2017, 8, 14542.

11 H. Robatjazi, et al., Plasmon-induced selective carbon dioxide conversion on earth-abundant aluminum-cuprous oxide antenna-reactor nanoparticles, Nat. Commun., 2017, 8, 27.

$12 \mathrm{~W}$. Xie and S. Schlücker, Hot electron-induced reduction of small molecules on photorecycling metal surfaces, Nat. Commun., 2015, 6, 7570.

13 Y. Kim, D. Dumett Torres and P. K. Jain, Activation Energies of Plasmonic Catalysts, Nano Lett., 2016, 16, 3399-3407.

14 G. Tagliabue, et al., Quantifying the role of surface plasmon excitation and hot carrier transport in plasmonic devices, Nat. Commun., 2018, 9, 3394.

15 M. J. Kale and P. Christopher, Plasmons at the interface, Science, 2015, 349, 587-588.

16 S. Chavez, U. Aslam and S. Linic, Design Principles for Directing Energy and Energetic Charge Flow in Multicomponent Plasmonic Nanostructures, ACS Energy Lett., 2018, 3, 1590-1596.

17 E. Kazuma, J. Jung, H. Ueba, M. Trenary and Y. Kim, Real-space and real-time observation of a plasmon-induced chemical reaction of a single molecule, Science, 2018, 360, 521-526. 
18 I. L. Violi, J. Gargiulo, C. von Bilderling, E. Cortés and F. D. Stefani, LightInduced Polarization-Directed Growth of Optically Printed Gold Nanoparticles, Nano Lett., 2016, 16, 6529-6533.

19 C. Boerigter, R. Campana, M. Morabito and S. Linic, Evidence and implications of direct charge excitation as the dominant mechanism in plasmon-mediated photocatalysis, Nat. Commun., 2016, 7, 10545.

$20 \mathrm{~K}$. Li, et al., Balancing Near-Field Enhancement, Absorption, and Scattering for Effective Antenna-Reactor Plasmonic Photocatalysis, Nano Lett., 2017, 17, 3710-3717.

$21 \mathrm{~K} . \mathrm{Wu}, \mathrm{J}$. Chen, J. R. McBride and T. Lian, Efficient hot-electron transfer by a plasmon-induced interfacial charge-transfer transition, Science, 2015, 349, 632-635.

22 E. Cortés, et al., Plasmonic hot electron transport drives nano-localized chemistry, Nat. Commun., 2017, 8, 14880.

23 C.-Y. Wu, et al., High-spatial-resolution mapping of catalytic reactions on single particles, Nature, 2017, 541, 511.

24 C. Zhan, et al., From plasmon-enhanced molecular spectroscopy to plasmonmediated chemical reactions, Nat. Rev. Chem., 2018, 2, 216-230.

25 L. Liu and A. Corma, Metal Catalysts for Heterogeneous Catalysis: From Single Atoms to Nanoclusters and Nanoparticles, Chem. Rev., 2018, 118, 4981-5079.

26 C. Novo, A. M. Funston and P. Mulvaney, Direct observation of chemical reactions on single gold nanocrystals using surface plasmon spectroscopy, Nat. Nanotechnol., 2008, 3, 598.

27 J. B. Sambur and P. Chen, Approaches to Single-Nanoparticle Catalysis, Annu. Rev. Phys. Chem., 2014, 65, 395-422.

28 W. Xu, J. S. Kong, Y.-T. E. Yeh and P. Chen, Single-molecule nanocatalysis reveals heterogeneous reaction pathways and catalytic dynamics, Nat. Mater., 2008, 7, 992.

$29 \mathrm{~W}$. Wang, Imaging the chemical activity of single nanoparticles with optical microscopy, Chem. Soc. Rev., 2018, 47, 2485-2508.

30 D. Nicolas-Debarnot and F. Poncin-Epaillard, Polyaniline as a new sensitive layer for gas sensors, Anal. Chim. Acta, 2003, 475, 1-15.

31 Y. Leroux, et al., Tunable Electrochemical Switch of the Optical Properties of Metallic Nanoparticles, ACS Nano, 2008, 2, 728-732.

32 T. Ding, J. Mertens, A. Lombardi, O. A. Scherman and J. J. Baumberg, LightDirected Tuning of Plasmon Resonances via Plasmon-Induced Polymerization Using Hot Electrons, ACS Photonics, 2017, 4, 1453-1458.

33 C. P. Byers, et al., Single-Particle Spectroscopy Reveals Heterogeneity in Electrochemical Tuning of the Localized Surface Plasmon, J. Phys. Chem. B, 2014, 118, 14047-14055.

34 K. A. Willets, A. J. Wilson, V. Sundaresan and P. B. Joshi, Super-Resolution Imaging and Plasmonics, Chem. Rev., 2017, 117, 7538-7582.

35 T. Chen, et al., Optical Super-Resolution Imaging of Surface Reactions, Chem. Rev., 2017, 117, 7510-7537.

36 S. Simoncelli, Y. Li, E. Cortés and S. A. Maier, Nanoscale Control of Molecular Self-Assembly Induced by Plasmonic Hot-Electron Dynamics, ACS Nano, 2018, 12, 2184-2192. 
37 S. Simoncelli, Y. Li, E. Cortés and S. A. Maier, Imaging Plasmon Hybridization of Fano Resonances via Hot-Electron-Mediated Absorption Mapping, Nano Lett., 2018, 18, 3400-3406.

38 R. Jungmann, et al., Multiplexed 3D cellular super-resolution imaging with DNA-PAINT and Exchange-PAINT, Nat. Methods, 2014, 11, 313.

39 P. Anger, P. Bharadwaj and L. Novotny, Enhancement and Quenching of Single-Molecule Fluorescence, Phys. Rev. Lett., 2006, 96, 113002.

40 T. M. Herne and M. J. Tarlov, Characterization of DNA Probes Immobilized on Gold Surfaces, J. Am. Chem. Soc., 1997, 119, 8916-8920.

41 X. Zhou, W. Xu, G. Liu, D. Panda and P. Chen, Size-Dependent Catalytic Activity and Dynamics of Gold Nanoparticles at the Single-Molecule Level, $J$. Am. Chem. Soc., 2010, 132, 138-146.

42 T. Chen, et al., Single-Molecule Nanocatalysis Reveals Facet-Dependent Catalytic Kinetics and Dynamics of Palladium Nanoparticles, ACS Catal., 2017, 7, 2967-2972.

43 T. Chen, Y. Zhang and W. Xu, Single-Molecule Nanocatalysis Reveals Catalytic Activation Energy of Single Nanocatalysts, J. Am. Chem. Soc., 2016, 138, 1241412421.

44 G. Baffou, R. Quidant and F. J. García de Abajo, Nanoscale Control of Optical Heating in Complex Plasmonic Systems, ACS Nano, 2010, 4, 709-716.

45 G. Baffou, R. Quidant and C. Girard, Thermoplasmonics modeling: A Green's function approach, Phys. Rev. B, 2010, 82, 165424.

46 J. H. Yoon, F. Selbach, L. Langolf and S. Schlücker, Ideal Dimers of Gold Nanospheres for Precision Plasmonics: Synthesis and Characterization at the Single-Particle Level for Identification of Higher Order Modes, Small, 2018, 14, 1702754.

47 S. Xing, et al., Highly controlled core/shell structures: tunable conductive polymer shells on gold nanoparticles and nanochains, J. Mater. Chem., 2009, 19, 3286-3291. 